\title{
Cardioactive Steroid Poisoning: A Comparison of Plant- and Animal-Derived Compounds
}

\author{
Fermin Barrueto, Jr., MD a, Barbara M. Kirrane, MD b, Brian W. Cotter, MS b, \\ Robert S. Hoffman, MD b, Lewis S. Nelson, MD ${ }^{\mathrm{b}}$
}

a Department of Emergency Medicine, University of Maryland School of Medicine and Department of Emergency Medicine, George Washington University

b New York City Poison Control Center, New York University-Bellevue Hospital Center, New York, NY

\begin{abstract}
Introduction: Cardioactive steroids (CASs) are found in plants, animals, and insects. Their affinity for Na+-K+ ATPase is attenuated by the type of lactone at carbon 17 (C17) of the steroid backbone: those with 5-membered lactone rings, or cardenolides, are derived mostly from plants with 6-membered rings or from animals with bufadienolides. A systematic review of CAS poisoning was performed to compare the mortality rate of cardenolides and bufadienolides.

Methods: MEDLINE was searched for articles using commonly reported names of CASs, and keywords were limited to human cases only. We searched cases from 1982 to 2003, so that supportive care was similar and digoxin-specific Fab was available. Identified reports of CAS poisoning were read to exclude cases involving licensed pharmaceuticals. Inclusion criteria included hyperkalemia, gastrointestinal symptoms, electrocardiographic evidence of CAS toxicity, digoxin serum concentration, or history of exposure to a substance containing a CAS. Clinical data was collected, including information about treatment with digoxin-specific Fab and treatment outcome.

Results: Fifty-nine articles, describing 924 patients, were identified. Eight hundred ninety-seven patients (97\%) ingested a CAS with a 5-membered lactone ring, and mortality was $6 \%(\mathrm{n}=54)$. Twenty-seven patients $(2.9 \%)$ ingested a CAS with a 6 -membered lactone ring, and mortality was $29.6 \%(\mathrm{n}=8)$. The difference in mortality rates was statistically significant $\left(\mathrm{p}<0.001,\left[\mathrm{X}^{2}\right]\right)$. CASs with 6-member rings accounted for the highest percentage of nonsuicidal exposures.

Conclusion: Although cardenolides accounted for the majority of exposures, bufadienolides were five times more lethal than cardenolides.
\end{abstract}

\section{INTRODUCTION}

The Ebers Papyrus (1552 BC), the oldest preserved medical document and perhaps first book of cardiology, suggests that Egyptians used plants that contained cardioactive steroids (CAS)
[1]. William Withering was the first modern author to describe the effects of foxglove to treat "dropsy," swelling of the body and presumed heart failure [2]. Today, digoxin and digitoxin, isolated from plants, are commonly used to treat congestive heart failure and atrial fibrillation.

Keywords: cardioactive steroids, cardenolides, bufadienolides, cardiac glycosides, digoxin

Acknowledgments: The authors thank Linda J. Kesselring, MS, ELS, medical copyeditor in the Department of Emergency Medicine at the University of Maryland School of Medicine, for her invaluable assistance with the preparation of this manuscript.

Notes: Presented at the North American Congress of Clinical Toxicologists, Chicago, Illinois, September 2003.

There was no outside funding of any kind used for this study.

Corresponding Author: Fermin Barrueto, Jr., MD, Division of Emergency Medicine, University of Maryland School of Medicine, 110 South Paca Street, 6th Floor, Suite 200, Baltimore, MD 21201. Email: fbarr001@umaryland.edu 


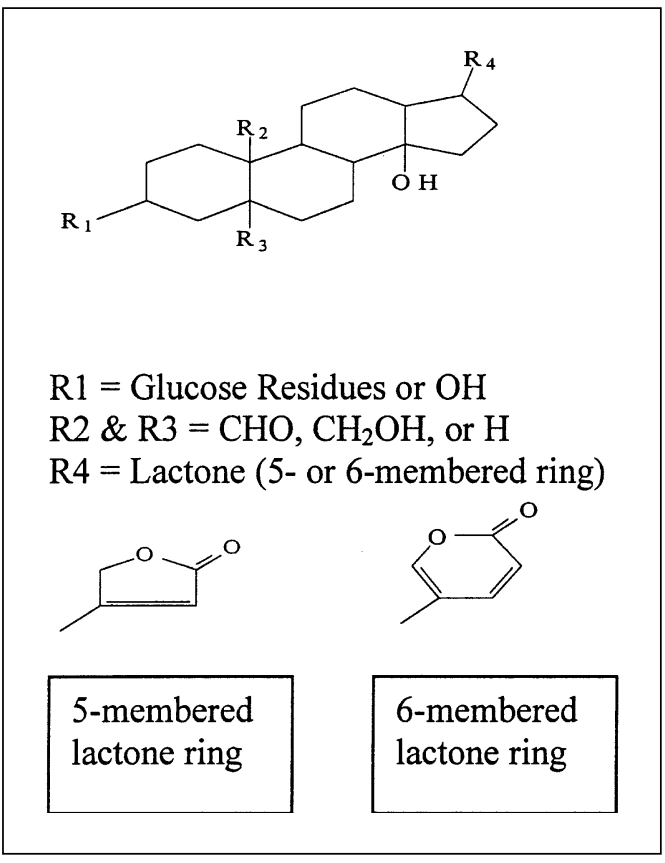

Figure 1. Cardioactive steroid backbone structure.

A CAS consists of a steroid nucleus and an unsaturated 5- or 6-membered lactone ring (Figure 1). Cardioactive steroids are classified as a cardenolide (with a 5-membered lactone ring) or a bufadienolide (with a 6-membered lactone ring). Additionally, substitution of a sugar residue on the $3 \beta-\mathrm{OH}$ group at $\mathrm{R} 1$ creates a cardiac glycoside (Figure 1).

Most cardenolides are derived from plants; for example, digitoxin and digoxin are extracted from the Digitalis genus (foxglove). In contrast, most bufadienolides, such as bufalin, are derived from mammals and amphibians (Figure 2). The bufadienolides also have an interesting insect source (the lightning bug, Photinus spp.) and a few plant sources; for example, red squill, Urginea maritima [3]. Human endogenous digoxin-like immunoreactive substance (EDLIS) is likely a combination of the cardenolide ouabain and bufadienolides such as marinobufagenin [4].
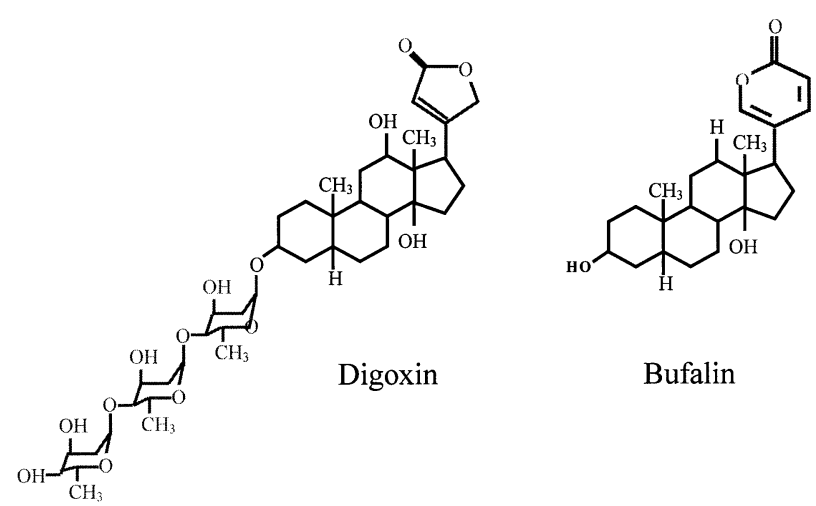

Bufalin
The clinical effects of regulated products such as digoxin and digitoxin are well known, and the signs and symptoms of overdose are well described. Herbal medicines and plants collected by foraging present a challenge for clinicians who must identify and treat CAS poisoning caused by nonpharmaceutical sources. These practices present the possibility of "mistaken identity," as when Digitalis lanata (containing digoxin) was mistaken for a plantain plant and incorporated into an herbal product. This product was mass produced and distributed in the United States, and it resulted in several cases of CAS poisoning [5]. In Sri Lanka, CAS poisoning caused by ingestion of yellow oleander (Thevetia peruviana) and its seeds are a common method of suicide [6-7]. In New York City, an epidemic of CAS poisoning occurred when a traditional Chinese aphrodisiac (known as Chan $\mathrm{Su}$, and commonly sold as "Love Stone") was ingested instead of being topically applied; the instructions explaining its topical use were, unfortunately, written only in Chinese [8]. Chan Su, derived from the secretions of the bufo toad (Bufo marinus), caused several reported deaths. These natural products contain CAS molecules that vary in structure, the defining portion being the lactone ring.

A multitude of nonpharmaceutical exposures to cardenolides and bufadienolides have been described in the literature, but the physiologic effects of these two groups have not been compared. The purpose of this review was to determine if a difference in mortality exists between these two major groups of cardioactive steroids.

\section{METHODS}

By searching MEDLINE from 1982 to 2003, a retrospective analysis for nonpharmaceutical human exposures to CASs was performed. This period was selected so that medical care was similar and digoxin-specific Fab (Digibind® or DigiFab®) was available. Keywords included any known herbal products that contain CAS, every genus and/or species of plant or animal known to contain CAS, as well as the names of specific CASs-namely bufalin, digoxin, and digitoxin (Table 1). These headings were interchanged with the word "herbal," and limits were placed on keywords so that only human cases were brought forward. Using a list contained within a review by Melero et al. of CAS, we constructed our list of CAS sources [9]. Papers published in languages other than English were translated. The full text of all reports identified by the MEDLINE search was collected, and two physicians reviewed the articles and needed to agree that each article met the requirement of CAS poisoning and did not describe licensed pharmaceuticals such as digoxin or digitoxin tablets. The articles' reference lists were similarly reviewed to identify any further cases. CAS poisoning was defined as containing any 3 of the following characteristics: 1) history of exposure to nonpharmaceutical substances containing CAS, 2) otherwise unexplained hyperkalemia, 3) gastrointestinal symptoms, 4) electrocardiographic evidence of digitalis toxicity, and 5) positive digoxin serum concentration. Two physicians reviewed each article for the five criteria, and the physicians needed to agree that an article

Figure 2. Digoxin, a cardenolide and cardiac glycoside. Bufalin is a bufadienolide. 


\section{TABLE 1. Search Terms for Cardioactive Steroids}

\begin{tabular}{|c|c|}
\hline Acokanthera & Helleborus \\
\hline Adenium & Herbal Digoxin \\
\hline Adonis & Herbal Tea \\
\hline Antiaria & Herbal \\
\hline Apocynum & Homeria \\
\hline Ascliepias & Kalanchoe \\
\hline Bowiea & Kyushin \\
\hline Bufalin & Lilly of the Valley \\
\hline Bufo (dienolide) & Love Stone \\
\hline Calotropis & Lucibufagin \\
\hline Cardiac glycoside & Moraea \\
\hline Cerbera & Oleander \\
\hline Chan Su & Oleandrogenin \\
\hline Cheiranthus & Periploca \\
\hline Convallaria & Photinus \\
\hline Coronilla & Squill \\
\hline Cotyledon & Strophanthus \\
\hline Cryptostegia & Thesium \\
\hline Digitalis (spp.) & Thevetia \\
\hline Erysimum & Toad Venom \\
\hline Erythrophleum & Tylecodon \\
\hline Euonymus & Urginea \\
\hline Gomphocarpus & Xysmalobium \\
\hline
\end{tabular}

met the criteria for inclusion. These reviewers were not blinded and were involved with the data collection. Clinical data were collected, including treatment with digoxin-specific Fab and the outcome of clinical treatment.

Using chi-squared analysis or Fisher's Exact Test, as applicable, we compared overall mortality and mortality associated with cardenolide- and bufadienolide-induced poisoning treated with digoxin-specific Fab. We also looked at the proportion of intentional exposures (attempted or fatal suicides).

\section{RESULTS}

Fifty-nine relevant articles were identified, describing a total of 924 patients whose reported clinical presentations met the inclusion criteria. The reviewers agreed, 100\%, that these articles contained at least 3 of the 5 criteria needed to meet the definition of CAS poisoning.

The overall mortality rate associated with nonpharmaceutical CAS exposures was $6.7 \%$ (62/924). The mortality among the 897 patients (97\% of the study group) who ingested a cardenolide was $6 \%$ [95\% confidence interval (CI) $4.64 \%-7.77 \%], \mathrm{n}=54$. The mortality among the 27 patients (3\% of the study group) who ingested a bufadienolide was $29.6 \%$ [95\% CI $15.85 \%-48.48 \%$ ], $\mathrm{n}=8$. The difference in mortality rates was statistically significant $\left(\mathrm{p}<0.001, \mathrm{X}^{2}\right)$ and the relative risk was 4.9218 [95\% CI $2.6051-$ .2988 ]. Seven percent [ $95 \%$ CI $2.06 \%-23.37 \%$ ], $n=2$, of the bufadienolide ingestions were suicide attempts compared to the 94\% [95\% CI 92.60 - 95.65\%], $\mathrm{n}=846$, of the cardenolide ingestions ( $p<0.001$, Fisher's Exact Test). Seven percent $(63 / 924)$ of all cases were treated with digoxin-specific Fab. Eight percent $[95 \%$ CI $3.49 \%-17.53 \%$ ] (5/62) of the patients treated with digoxinspecific Fab died after ingesting either type of CAS compared to the $6.7 \%(58 / 862)$ [95\% CI 5.24\% - 8.60\%] of patients who did not receive treatment $\left(\mathrm{p}=\mathrm{NS}, \mathrm{X}^{2}\right)$.

\section{DISCUSSION}

Plant and animal based CASs contain various molecular combinations at R1, R2, and R3 (Figure 1); however, the one constant within each category is the R4 substitution, which remains the same within its respective source. In our review of bufadienolides and cardenolides, mortality rate was 5-fold higher when exposure involved a bufadienolide. However, variables such as dose and absorption were not addressed and impossible to determine with the data collected because there is no laboratory analysis that quantifies the amount of CAS when it is a mixture. The most common preparation that contained bufadienolides was the Chinese aphrodisiac Chan Su ("Love Stone"). A specific analysis of this preparation and comparison to oleander, the most common plant source of CAS poisoning, would provide further insight.

The plant sources of CAS are much more varied than the animal sources and are more often described in the literature. The most common are Digitalis lanata, Digitalis purpurea, Nerium oleander, and Thevetia peruviana. The most common animal source of CAS exposure is the bufo toad. Interestingly, herbal medications prescribed for "heart" patients by some herbalists specify bufo toad venom, such as kyushin, as an ingredient [10].

Although created to bind digoxin, digoxin-specific Fab binds a variety of CASs, including cardenolides such as oleandrogenin and bufadienolides such as bufalin and cinobufatolin [11]. In our retrospective analysis, treatment with digoxin-specific Fab did not appear to alter the mortality rate, but only $7 \%$ of patients were treated with digoxin-specific Fab. In addition, some of the published reports came from countries where digoxin-specific Fab was not available as a treatment option. Given the retrospective and uncontrolled nature of the study, no conclusions can be made about response to Fab. In a prospective study of nonpharmaceutical CAS poisoning, Eddleston et al. found that digoxinspecific Fab decreased mortality by half when administered to patients exposed to yellow oleander (Thevetia peruviana) [12]. Their study supported what had been suggested in vitro and in animal models: digoxin-specific Fab cross-reacts with cardenolides and bufadienolides to treat patients effectively $[11,13]$.

A health care provider should suspect a nonpharmaceutical CAS exposure when a patient, after ingesting a known or unidentified 
product, presents with nausea, vomiting, abdominal pain, unexplained hyperkalemia, and electrocardiogram findings consistent with digoxin toxicity. In these cases, digoxin-specific Fab should be considered antidotal therapy and administered as appropriate. Heightened caution may be warranted if the patient is known to use an herbal product that contains bufadienolides, given the 5-fold increase in mortality revealed by our review. Further analysis and study of these animal, plant, and herbal sources of CAS is needed to assess the actual dose contained in these substances.

\section{LIMITATIONS}

This study carries all the limitations of a retrospective literaturebased analysis, including the potential for reporting bias and the generally poor documentation of clinical effects; however, a prospective study of nonpharmaceutical CAS exposures in humans would be difficult to perform. PubMed was the only searched database. Further searches of alternative medicine databases and ToxNet should be performed. In general, the reported cases did not provide specific identification or analysis of the CAS source or the analysis of serum concentrations of the various CASs-either to confirm exposure or to provide the basis for a dose-toxicity relationship. This makes it difficult to specify if bufadienolides are more toxic than cardenolides. There was nonstandardized treatment for these patients and an inability to account for co-ingestants in this review. Also, since cases that are unique or result in death are more likely to be reported in the literature (reporting bias), the actual overall mortality rate may be lower than reported. However, a prospective study that focused on yellow oleander (a CAS with a 5-membered lactone ring) found a similar mortality rate of 5\% to $10 \%$ [14].

\section{CONCLUSION}

Physicians should be aware of the various ways people are exposed to nonpharmaceutical cardioactive steroids. The cardenolides (generally plant-derived compounds) are more plentiful and are more likely to be involved in a CAS exposure. Bufodienolides (generally animal-based products) are less available but result in a mortality rate that is five times greater than cardenolides.

The authors have no potential conflicts of interest to report.

\section{REFERENCES}

1. Ziskind B, Halioua B. Contribution of Ancient Egypt to cardiovascular medicine. Arch Mal Coeur Vaiss 2004;97(4): 370-374.
2. Wade OL. Digoxin 1785-1985. I. Two hundred years of digitalis. J Clin Hosp Pharm 1986;11(1):3-9.

3. Eisner T, Goetz MA, Hill DE, Smedley SR, Meinwald J. Firefly "femmes fatales" acquire defensive steroids (lucibufagins) from their firefly prey. Proc Natl Acad Sci USA 1997;94(18):9723-9728.

4. Schoner W. Endogenous cardiac glycosides, A new class of steroid hormones. Eur J Biochem 2002;269(10):2440-2448.

5. Slifman NR, Obermeyer WR, Aloi BK, Musser SM, Correll WA Jr, Cichowicz SM, Betz JM, Love LA. Contamination of botanical dietary supplements by Digitalis lanata. N Engl J Med 1998;339(12):806-811.

6. Eddleston M, Ariaratnam CA, Sjostrom L, Jayalath S, Rajakanthan K, Rajapakse S, Colbert D, Meyer WP, Perera G, Attapattu S, Kularatne SA, Sheriff MR, Warrell DA. Acute yellow oleander (Thevetia peruviana) poisoning: cardiac arrhythmias, electrolyte disturbances, and serum cardiac glycoside concentrations on presentation to hospital. Heart

2000;83(3):301-306.

7. Eddleston M, Ariaratnam CA, Meyer WP, Perera G, Kularatne AM, Attapattu S, Sheriff MH, Warrell DA. Epidemic of self-poisoning with seeds of the yellow oleander tree (Thevetia peruviana) in northern Sri Lanka. Trop Med Int Health 1999;4(4):266-273.

8. Brubacher JR, Ravikumar PR, Bania T, Heller MB, Hoffman RS. Treatment of toad venom poisoning with digoxin-specific Fab fragments. Chest 1996;110(5):1282-1288.

9. Melero CP, Medarde M, San Felician F. A short review on Cardiotonic Steroids and Their Aminoguanidine Analgues.

Molecules 2000;5:51-81.

10. Lin CS, Lin MC, Chen KS, Ho CC, Tsai SR, Ho CS, Shieh $\mathrm{WH}$. A digoxin-like immunoreactive substance and atrioventricular block induced by a Chinese medicine "kyushin". Jpn Circ J 1989;53(9):1077-1080.

11. Dasgupta A, Emerson L. Neutralization of cardiac toxins oleandrin, oleandrigenin, bufalin, and cinobufotalin by digibind: Monitoring the effect by measuring free digitoxin concentrations. Life Sci 1998;63(9):781-788.

12. Eddleston M, Senarathna L, Mohamed F, Buckley N, Juszczak E, Sheriff MH, Ariaratnam A, Rajapakse S, Warrell D, Rajakanthan K. Deaths due to absence of an affordable antitoxin for plant poisoning. Lancet 2003;362(9389): 1041-1044.

13. Brubacher JR, Lachmanen D, Ravikumar PR, Hoffman RS. Efficacy of digoxin specific Fab fragments (Digibind) in the treatment of toad venom poisoning. Toxicon 1999;37(6):931-942.

14. Eddleston M, Warrell DA. Management of acute yellow oleander poisoning. QJM 1999;92(9):483-485. 